\title{
On the vibrations of a mechanically based non-local beam model
}

\author{
Mario Di Paola ${ }^{a}$, Giuseppe Failla ${ }^{\mathrm{b}}$, Alba Sofi ${ }^{\mathrm{b}, *}$, Massimiliano Zingales ${ }^{\mathrm{a}}$ \\ ${ }^{a}$ Dipartimento di Ingegneria Civile, Ambientale e Aerospaziale (DICA), Università di Palermo, Viale delle Scienze, 90128 Palermo, Italy \\ ${ }^{\mathrm{b}}$ Dipartimento di Meccanica e Materiali (MECMAT), Università Mediterranea di Reggio Calabria, Via Graziella, Località Feo di Vito, 89122 Reggio Calabria, Italy
}

\section{A R T I C L E I N F O}

\section{Article history:}

Received 1 November 2011

Received in revised form 14 March 2012

Accepted 15 March 2012

Available online 11 April 2012

\section{Keywords:}

Non-local elasticity

Long-range interactions

Timoshenko beam theory

Hamilton's principle

Free vibrations

\begin{abstract}
A B S T R A C T
The vibration problem of a Timoshenko non-local beam is addressed. The beam model involves assuming that the equilibrium of each volume element is attained due to contact forces and long-range body forces exerted, respectively, by adjacent and non-adjacent volume elements. The contact forces result in the classical Cauchy stress tensor while the long-range forces are taken as depending on the product of the interacting volume elements and on their relative displacement through a material-dependent distance-decaying function. To derive the motion equations and the related mechanical boundary conditions, the Hamilton's principle is applied.
\end{abstract}

(c) 2012 Elsevier B.V. All rights reserved.

\section{Introduction}

The classical beam theories have been recently reinterpreted in a non-local framework. Besides its theoretical interest, this effort has been certainly motivated by the increasing importance of nanobeams in electromechanical system devices, such as sensors and actuators, where small-size effects play a crucial role, as shown by experimental evidence [1,2] and atomistic simulations [3]. In this context, non-local beam theories have been soon preferred to classical local beam theories, where the influence of microstructure cannot be brought into account, as well as to very demanding atomistic simulations.

In general, the non-local beam theories developed to date have concerned linearly elastic behavior. In the first studies, non-local effects have been modeled based on the Eringen's integral theory, according to which the stress at a given point depends on the strain in the whole beam volume. The correct framework to derive the non-local stress resultants has been built by Lu and co-workers [4]. They showed that, in general, the natural frequencies of both the Euler-Bernoulli (EB) and the Timoshenko (TM) beam tend to decrease due to non-local effects, although the opposite behavior is exhibited by the first natural frequency of a EB cantilever beam [4]. Later, Reddy [5] showed that in a simply-supported beam the natural frequencies decrease due to non-local effects not only when the EB or the TM theories are used, but also for alternative beam theories.

\footnotetext{
* Corresponding author. Tel.: +39 0965 875225; fax: +39 0965875201

E-mail address: alba.sofi@unirc.it (A. Sofi).
}

In recent years, non-local theories alternative to the classical Eringen's theory have been also used to formulate the vibration problem of non-local beams. For instance, Zhang et al. [6] derived the motion equations of a EB beam by the Hamilton's principle used in conjunction with a so-called hybrid approach, which involves assuming that the strain energy functional depends on local and non-local curvatures. The natural frequencies have been found to decrease as a result of non-local effects, but to a smaller extent with respect to those predicted by the classical Eringen's theory. Lakes [7] have used the classical EB theory in conjunction with a continuum micropolar elasticity constitutive law, to be interpreted as the result of long-range forces mutually exerted by non-nearest neighbor atoms. Kong et al. [8] derived the motion equations of a TM non-local beam by the Hamilton's principle, based on a modified couple stress theory. Wang et al. [9] also used the Hamilton's principle but in conjunction with the gradient elasticity theory proposed in Ref. [1]. All the studies in Refs. [2,8,9] showed that non-local effects determine an increase in the natural frequencies of the proposed non-local beam, a result that matches experimental evidence of polypropylene and epoxy micro-cantilever beams $[1,2]$.

The foregoing discussion suggests that the non-local beam models developed to date may lead to quite different predictions on the dynamic response, depending on the beam theory (EB or TM theory) and on the modeling of non-local effects. Certainly, it is expected that a progressive refinement of the theoretical models will be possible as more experimental evidence will be gained. However, in view of the complexity and variety of different microstructures that may be encountered in practical applications and considering the increasing number of new materials that is likely 
to be produced in the future, in the authors' opinion new theoretical models, that may fit a variety of material behaviors by setting a sufficient but relatively limited number of parameters, are certainly highly desirable.

To this purpose, this paper will present the dynamics of a TM non-local beam model cast within a mechanically based approach to non-local elasticity recently proposed by the authors [10-12].

\section{Mechanically based non-local elasticity}

A detailed description of the mechanically based approach to non-local elasticity theory recently proposed by the authors can be found in Refs. [10-12]. Here the key concepts useful for the later developments will be summarized.

Consider a continuum of volume $V$ and boundary surface $S=S_{c} \cup S_{f}\left(S_{c}\right.$ and $S_{f}$ are the constrained and unconstrained parts) referred to a rectangular Cartesian coordinate system $O(x, y, z)$. The non-local effects are modeled as long-range internal body forces exchanged between non-adjacent elementary volume elements. Specifically, they are built as central forces proportional to the interacting volume elements and to their relative displacement through a distance-decaying attenuation function. The resultant of the long-range forces exerted on the unit volume at $\mathbf{x}=\left[\begin{array}{lll}x y z & z\end{array}\right]^{T}$ by all the volumes $d V(\xi)$ at $(\xi)=[\xi \psi \zeta]^{T}$ is given as

$\mathbf{f}(\mathbf{x})=\int_{V} \mathbf{q}(\mathbf{x}, \xi) d V(\boldsymbol{\xi})=\int_{V} g(\mathbf{x}, \xi)\left[\boldsymbol{\eta}^{T}(\mathbf{x}, \xi) \mathbf{r}(\mathbf{x}, \xi)\right] \mathbf{r}(\mathbf{x}, \xi) d V(\xi)$

where $\mathbf{q}(\mathbf{x}, \boldsymbol{\xi})=g(\mathbf{x}, \boldsymbol{\xi})\left[\boldsymbol{\eta}^{T}(\mathbf{x}, \boldsymbol{\xi}) \mathbf{r}(\mathbf{x}, \boldsymbol{\xi})\right] \mathbf{r}(\mathbf{x}, \boldsymbol{\xi})$ denotes the (specific) central long-range force exerted on a unit volume at $\mathbf{x}$ by a unit volume at $\boldsymbol{\xi}$, being $\mathbf{r}(\mathbf{x}, \boldsymbol{\xi})=\left[\begin{array}{lll}r_{x} & r_{y} & r_{z}\end{array}\right]^{T}$ the unit vector associated with the direction $\mathbf{x}-\boldsymbol{\xi}$, positively oriented from $\mathbf{x}$ to $\xi ; g(\mathbf{x}, \boldsymbol{\xi})$ is a material-dependent, symmetric and distance-decaying real-valued scalar function, i.e. $g(\mathbf{x}, \boldsymbol{\xi})=g(\xi, \mathbf{x})$ and $g\left(\mathbf{x}_{1}, \boldsymbol{\xi}_{1}\right)<g\left(\mathbf{x}_{2}, \xi_{2}\right) \forall$ $\left|\mathbf{x}_{1}-\xi_{1}\right|>\left|\mathbf{x}_{2}-\xi_{2}\right| ; \boldsymbol{\eta}(\mathbf{x}, \xi)$ is the vector of the relative displacements between the centroids of the volumes $d V(\mathbf{x})$ and $d V(\xi)$, i.e. $\boldsymbol{\eta}(\mathbf{x}, \xi)=\mathbf{u}(\xi)-\mathbf{u}(\mathbf{x})$.

In the context of the proposed non-local theory, the longrange internal body forces $\mathbf{f}(\mathbf{x}) d V(\mathbf{x})$ contribute to the equilibrium of a volume $d V(\mathbf{x})$ along with the external body forces $\overline{\mathbf{b}}(\mathbf{x}) d V(\mathbf{x})$, and the classical contact Cauchy stresses $\boldsymbol{\sigma}^{(l)}(\mathbf{x})=$ $\left[\begin{array}{llllll}\sigma_{x}^{(l)} & \sigma_{y}^{(l)} & \sigma_{z}^{(l)} & \tau_{y z}^{(l)} & \tau_{x z}^{(l)} & \tau_{x y}^{(l)}\end{array}\right]^{T}$ where the superscript means local. Both $\mathbf{f}(\mathbf{x}) d V(\mathbf{x})$ and $\overline{\mathbf{b}}(\mathbf{x}) d V(\mathbf{x})$ are infinitesimals of the same order and thus the mechanical boundary conditions (BC) coincide with the classical BC, that is $\overline{\mathbf{p}}_{n}(\mathbf{x})=\mathbf{N} \boldsymbol{\sigma}^{(l)}(\mathbf{x})$ on $S_{f}$, being $\overline{\mathbf{p}}_{n}(\mathbf{x})$ the external surface load and $\mathbf{N}$ the boundary equilibrium operator involving the outward normal $\mathbf{n}$ to $S_{f}$.

For a linearly elastic and isotropic material the stress vector $\boldsymbol{\sigma}^{(l)}(\mathbf{x})$ is related to the vector $\boldsymbol{\varepsilon}(\mathbf{x})$ of the small strain components, i.e. $\boldsymbol{\varepsilon}(\mathbf{x})=\left[\begin{array}{llllll}\varepsilon_{x} & \varepsilon_{y} & \varepsilon_{z} & \gamma_{y z} & \gamma_{x z} & \gamma_{x y}\end{array}\right]^{T}$, by the local law

$$
\boldsymbol{\sigma}^{(l)}(\mathbf{x})=\mathbf{D}^{*} \boldsymbol{\varepsilon}(\mathbf{x}), \text { for } \mathbf{D}^{*}
$$

$$
=\left[\begin{array}{cccccc}
\left(2 \mu^{*}+\lambda^{*}\right) & \lambda^{*} & \lambda^{*} & 0 & 0 & 0 \\
\lambda^{*} & \left(2 \mu^{*}+\lambda^{*}\right) & \lambda^{*} & 0 & 0 & 0 \\
\lambda^{*} & \lambda^{*} & \left(2 \mu^{*}+\lambda^{*}\right) & 0 & 0 & 0 \\
0 & 0 & 0 & \mu^{*} & 0 & 0 \\
0 & 0 & 0 & 0 & \mu^{*} & 0 \\
0 & 0 & 0 & 0 & 0 & \mu^{*}
\end{array}\right] .
$$

In Eq. (2) $\mu^{*}=\beta_{1} \mu$ and $\lambda^{*}=\beta_{1} \lambda$, being $\mu$ and $\lambda$ the Lamè elastic constants and being $\beta_{1}$ a dimensionless real coefficient, $0 \leqslant \beta_{1} \leqslant 1$, weighting the amount of local interactions [13]. Furthermore, the strain-displacement equations $\boldsymbol{\varepsilon}(\mathbf{x})=\mathbb{C} \mathbf{u}(\mathbf{x})$ hold, being $\mathbb{C}$ the compatibility operator and $\mathbf{u}(\mathbf{x})$ the displacement vector, to which the kinematic BC $\mathbf{u}(\mathbf{x})=\overline{\mathbf{u}}(\mathbf{x})$, for $\mathbf{x} \in S_{c}$, apply.
As outlined in Ref. [10,11], the non-local model admits a consistent variational formulation based on the total potential elastic energy $\Pi(\mathbf{u}, \boldsymbol{\varepsilon})=\Phi(\mathbf{u}, \boldsymbol{\varepsilon})-W(\mathbf{u})$, where $\Phi(\mathbf{u}, \boldsymbol{\varepsilon})$ is the elastic potential energy and $W(\mathbf{u})$ is the work done by the external forces $\overline{\mathbf{b}}(\mathbf{x})$ and $\overline{\mathbf{p}}_{n}(\mathbf{x})$, being $\mathbf{u}(\mathbf{x})$ and $\boldsymbol{\varepsilon}(\mathbf{x})$ arbitrary functions satisfying the small strain-displacement equations and the kinematic BC $\mathbf{u}(\mathbf{x})=\overline{\mathbf{u}}(\mathbf{x})$ on $S_{c}$. The elastic potential energy $\Phi(\mathbf{u}, \boldsymbol{\varepsilon})$ is defined as

$$
\begin{aligned}
\Phi(\mathbf{u}, \boldsymbol{\varepsilon})= & \Phi^{(l)}(\boldsymbol{\varepsilon})+\Phi^{(n l)}(\boldsymbol{\eta})=\frac{1}{2} \int_{V} \boldsymbol{\varepsilon}^{T}(\mathbf{x}) \mathbf{D}^{*} \boldsymbol{\varepsilon}(\mathbf{x}) d V(\mathbf{x}) \\
& +\frac{1}{4} \int_{V} \int_{V} g(\mathbf{x}, \xi)\left[\mathbf{r}^{T}(\mathbf{x}, \xi) \boldsymbol{\eta}(\mathbf{x}, \xi)\right]^{2} d V(\xi) d V(\mathbf{x}),
\end{aligned}
$$

where $\Phi^{(l)}(\boldsymbol{\varepsilon})$ and $\Phi^{(n l)}(\boldsymbol{\eta})$ are the local and non-local contributions $[10,11]$. To derive the motion equations under time-varying external loads, the Hamilton's functional $H=K-(\Phi-W)$ can be considered where, $K$ is the kinetic energy given by

$K=\frac{1}{2} \int_{V} \rho\left(\frac{\partial \mathbf{u}(\mathbf{x}, t)}{\partial t}\right)^{T}\left(\frac{\partial \mathbf{u}(\mathbf{x}, t)}{\partial t}\right) d V(\mathbf{x})$

with $\rho$ denoting the mass density of the body (assumed to be time independent).

\section{Dynamics of the non-local Timoshenko beam}

Within the framework of the mechanically based approach briefly outlined in Section 2, the authors have recently developed a non-local TM beam model and investigated its response under static loads [12]. The corresponding equations of motion will be derived next by applying the Hamilton's principle.

Consider the straight uniform beam of length $L$ in Fig. 1, where the $x$-axis is the centroidal axis, the $y$ - and $z$-axes are the neutral and symmetry axis of the cross-section, whose area is denoted by $A$. The beam is subjected to time-varying external forces per unit length denoted by $F_{x}(x, t)$ and $F_{z}(x, t)$. The displacement components $u_{x}, u_{y}$ and $u_{z}$ take the form

$u_{x}=u(x, t)-z \varphi(x, t) ; \quad u_{y}=0 ; \quad u_{z}=v(x, t)$,

where $u(x, t)$ and $v(x, t)$ are the $x$ - and $z$-displacements of the crosssection at a point $(x, 0,0) ; \varphi(x, t)$ denotes the rotation of the crosssection about the $y$-axis (positive if clockwise). The corresponding nonzero strain components are

$\varepsilon_{x}=\frac{\partial u_{x}}{\partial x}=\varepsilon(x, t)+z \chi(x, t) ; \quad \gamma_{x z}=\frac{\partial u_{x}}{\partial z}+\frac{\partial u_{z}}{\partial x}=\gamma(x, t)$,

where $\varepsilon(x, t)=\partial u(x, t) / \partial x, \quad \chi(x, t)=-\partial \varphi(x, t) / \partial x, \quad \gamma(x, t)=\partial v(x, t) /$ $\partial x-\varphi(x, t)$ denote the generalized axial, bending and transverse shear strain, respectively. The latter are related to the corresponding stress resultants by the following constitutive laws:

$$
\begin{aligned}
& N^{(l)}(x, t)=\int_{A} \sigma_{x}^{(l)}(x, t) d A=E^{*} A \varepsilon(x, t) ; \\
& M^{(l)}(x, t)=\int_{A} z \sigma_{x}^{(l)}(x, t) d A=E^{*} I \chi(x, t) ; \\
& T^{(l)}(x, t)=\int_{A} \tau_{x z}^{(l)}(x, t) d A=K_{s} G^{*} A \gamma(x, t),
\end{aligned}
$$

where $N^{(l)}(x, t), T^{(l)}(x, t)$ and $M^{(l)}(x, t)$ are the axial force, the shear force and the bending moment of the classical local beam theory (see Fig. 1), $E^{*}=\beta_{1} E$ and $G^{*}=\beta_{1} G$, being $E$ and $G$ the Young's and shear modulus, respectively, while $\beta_{1}$ has been introduced in Section 2. Also, $I$ denotes the second moment of area about the $y$-axis and $K_{s}$ is the shear correction factor.

Upon introducing the constitutive relations (7) and taking into account Eqs. (5), the elastic potential energy (3) reads [12]: 

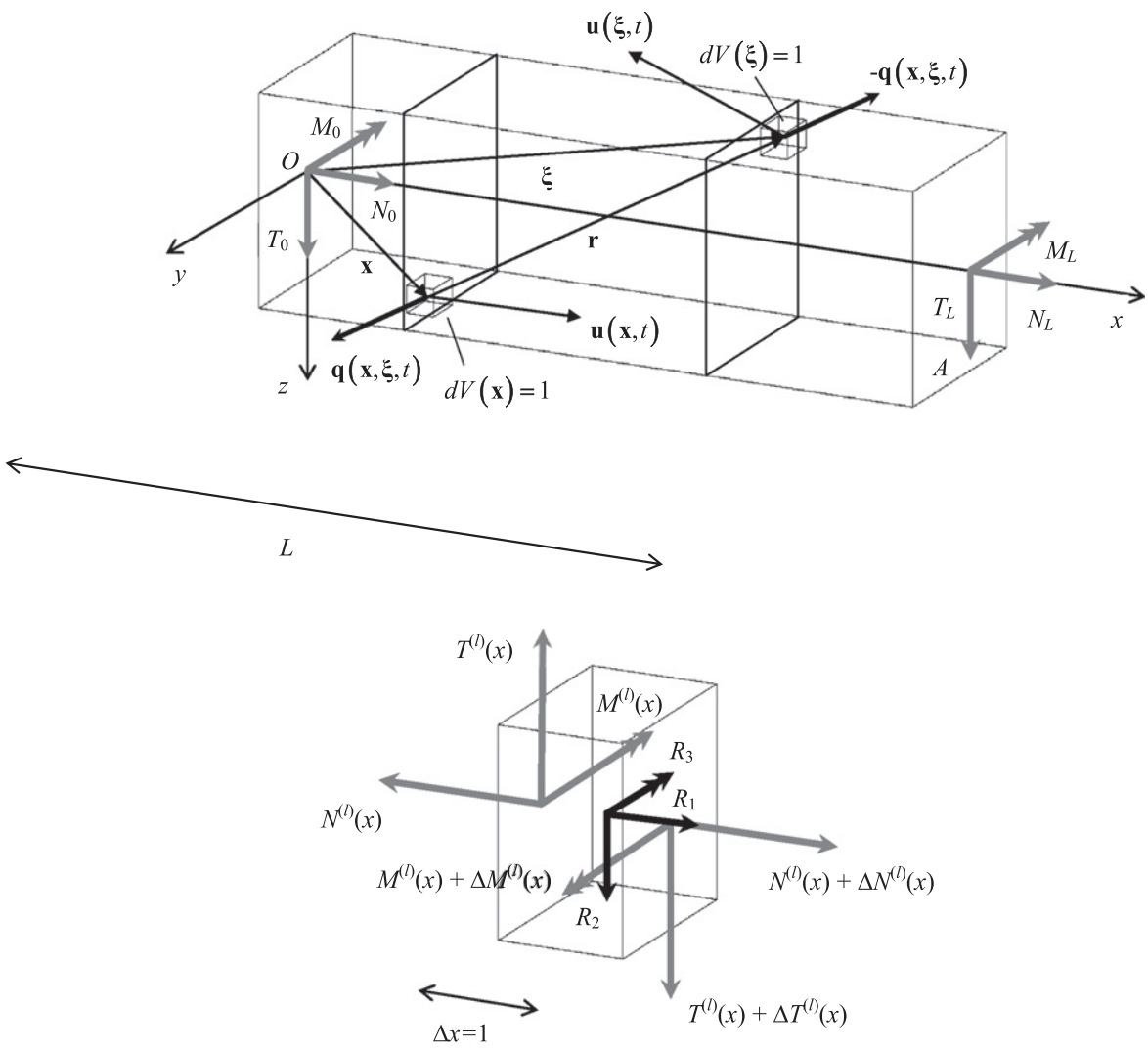

Fig. 1. Non-local beam model with long-range forces between non-adjacent volume elements.

$$
\begin{aligned}
\Phi\left(\varepsilon, \chi, \gamma, \eta_{x}, \eta_{z}\right)= & \Phi^{(l)}(\varepsilon, \chi, \gamma)+\Phi^{(n l)}\left(\eta_{x}, \eta_{z}\right) \\
= & \frac{1}{2} \int_{0}^{L}\left[E^{*} A \varepsilon^{2}+E^{*} I \chi^{2}+K_{s} G^{*} A \gamma^{2}\right] d x+\frac{1}{4} \int_{V} \\
& \times \int_{V}\left[q_{x} \eta_{x}+q_{z} \eta_{z}\right] d V(\mathbf{x}) d V(\xi),
\end{aligned}
$$

where $\eta_{x}$ and $\eta_{z}$ are the components of the relative displacement vector between the centroids of the elementary volumes $d V(\mathbf{x})$ and $\quad d V(\xi), \quad$ i.e. $\quad \eta_{x}=[u(\xi, t)-u(x, t)]-[\zeta \varphi(\xi, t)-z \varphi(x, t)]$, $\eta_{z}=v(\xi, t)-v(x, t)$, while $\eta_{y}=0$. Further, in Eq. (8) $q_{x}$ and $q_{z}$ are the time-dependent components of the specific long-range force in Eq. (1), i.e. (see Fig. 1)

$$
\begin{aligned}
q_{x}(\mathbf{x}, \xi, t)= & g(\mathbf{x}, \xi)\left\{[u(\xi, t)-u(x, t)] r_{x}^{2}(\mathbf{x}, \xi)-[\zeta \varphi(\xi, t)\right. \\
& -z \varphi(x, t)] r_{x}^{2}(\mathbf{x}, \xi)+[v(\xi, t) \\
& \left.-v(x, t)] r_{x}(\mathbf{x}, \xi) r_{z}(\mathbf{x}, \xi)\right\} \\
q_{z}(\mathbf{x}, \xi, t)= & g(\mathbf{x}, \xi)\left\{[u(\xi, t)-u(x, t)] r_{x}(\mathbf{x}, \xi) r_{z}(\mathbf{x}, \xi)\right. \\
& -[\zeta \varphi(\xi, t)-z \varphi(x, t)] r_{x}(\mathbf{x}, \xi) r_{z}(\mathbf{x}, \xi)+[v(\xi, t) \\
& \left.-v(x, t)] r_{z}^{2}(\mathbf{x}, \xi)\right\}
\end{aligned}
$$

where $r_{x}(\mathbf{x}, \xi), r_{y}(\mathbf{x}, \xi)$ and $r_{z}(\mathbf{x}, \xi)$ are the components of $\mathbf{r}(\mathbf{x}, \xi)$. Also, $W$ and $K$ can be cast as

$W=\int_{0}^{L}\left[F_{x} u+F_{z} v\right] d x+\sum_{i=0, L}\left[N_{i} u\left(x_{i}, t\right)+T_{i} v\left(x_{i}, t\right)+M_{i} \varphi\left(x_{i}, t\right)\right]$,

$K=\frac{1}{2} \int_{0}^{L} m\left[\left(\frac{\partial u}{\partial t}-z \frac{\partial \varphi}{\partial t}\right)^{2}+\left(\frac{\partial v}{\partial t}\right)^{2}\right] d x$

where $N_{i}, T_{i}$ and $M_{i}(i=0, L)$ are the axial force, transverse force and bending moment at $x_{i}=0, L, m$ is the mass per unit length.
The Hamilton's principle states that, among all the admissible dynamic paths, the actual motion of the beam with prescribed configurations at the time instants $t=0$ and $t=T$, in the interval $0<x<L$, satisfies the stationarity condition:

$\delta H=\delta \int_{0}^{T}[K-(\Phi-W)] d t=0$.

In light of the arbitrariness of $\delta u, \delta v$ and $\delta \varphi$ in the interval $0<x<L$, the first variation of the Hamilton's functional (12) yields the EulerLagrange equations

$$
\begin{aligned}
& E^{*} A \frac{\partial^{2} u(x, t)}{\partial x^{2}}+R_{1}(x, t)+F_{x}(x, t)=m \frac{\partial^{2} u(x, t)}{\partial t^{2}}, \\
& K_{s} G^{*} A\left[\frac{\partial^{2} v(x, t)}{\partial x^{2}}-\frac{\partial \varphi(x, t)}{\partial x}\right]+R_{2}(x, t)+F_{z}(x, t)=m \frac{\partial^{2} v(x, t)}{\partial t^{2}} \\
& E^{*} I \frac{\partial^{2} \varphi(x, t)}{\partial x^{2}}+K_{s} G^{*} A\left[\frac{\partial v(x, t)}{\partial x}-\varphi(x, t)\right]+R_{3}(x, t)=I_{\rho} \frac{\partial^{2} \varphi(x, t)}{\partial t^{2}}
\end{aligned}
$$

where $I_{\rho}=\int_{A} \rho z^{2} d A$, while $R_{1}(x, t), R_{2}(x, t)$ and $R_{3}(x, t)$ are the resultants per unit length of the long-range forces (see Fig. 1). If the cross-section has two axes of symmetry, such resultants are given by:

$$
\begin{aligned}
& R_{1}(x, t)=\int_{0}^{L} I_{1}(x, \xi)[u(\xi, t)-u(x, t)] d \xi \\
& R_{2}(x, t)=\int_{0}^{L}\left\{-I_{2}(x, \xi) \varphi(\xi, t)+I_{3}(x, \xi) \varphi(x, t)+I_{4}(x, \xi)[v(\xi, t)-v(x, t)]\right\} d \xi, \\
& R_{3}(x, t)=\int_{0}^{L}\left\{I_{5}(x, \xi) \varphi(\xi, t)-I_{6}(x, \xi) \varphi(x, t)-I_{3}(x, \xi)[v(\xi, t)-v(x, t)]\right\} d \xi
\end{aligned}
$$

with $I_{i}(x, \xi)(i=1,2, \ldots, 6)$ being the integrals defined in Ref. [12], not reported for brevity. The mechanical and kinematic BC associated to Eqs. (13) read 


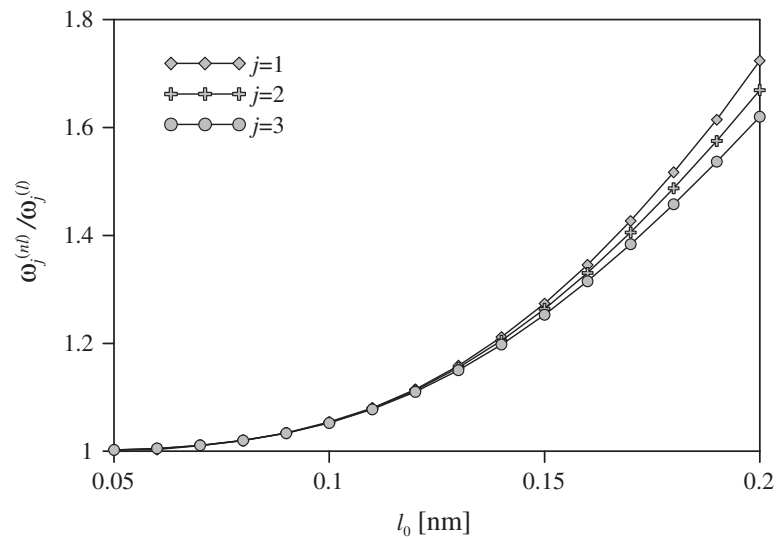

Fig. 2. First three natural frequencies of the simply-supported non-local beam normalized to the corresponding local values for different internal lengths $l_{0}$.

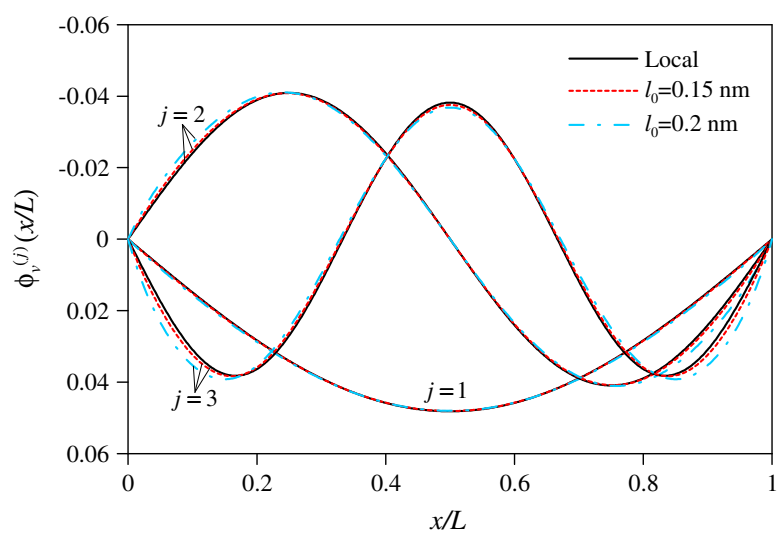

Fig. 3. First three vibration modes of the simply-supported non-local beam for different internal lengths $l_{0}$.

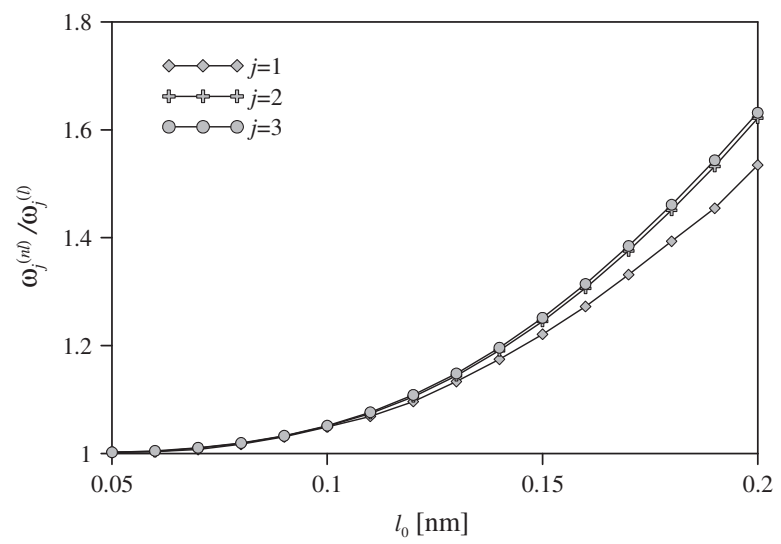

Fig. 4. First three natural frequencies of the cantilever non-local beam normalized to the corresponding local values for different internal lengths $l_{0}$.

$$
\begin{array}{r}
N^{(l)}\left(x_{i}, t\right)=\left.E^{*} A \frac{\partial u(x, t)}{\partial x}\right|_{x_{i}}=\mp N_{i} \text { or } \quad u\left(x_{i}, t\right)=u_{i}(t) \\
T^{(l)}\left(x_{i}, t\right)=\left.K_{s} G^{*} A\left[\frac{\partial v(x, t)}{\partial x}-\varphi(x, t)\right]\right|_{x_{i}}=\mp T_{i} \quad \text { or } \quad v\left(x_{i}, t\right)=v_{i}(t) \\
M^{(l)}\left(x_{i}, t\right)=-\left.E^{*} I \frac{\partial \varphi(x, t)}{\partial x}\right|_{x_{i}}= \pm M_{i} \text { or } \quad \varphi\left(x_{i}, t\right)=\varphi_{i}(t) .
\end{array}
$$

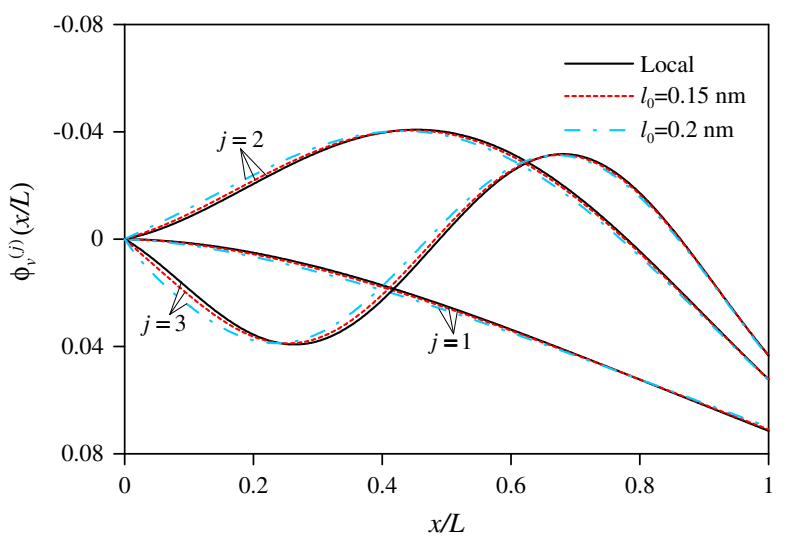

Fig. 5. First three vibration modes of the cantilever non-local beam for different internal lengths $l_{0}$

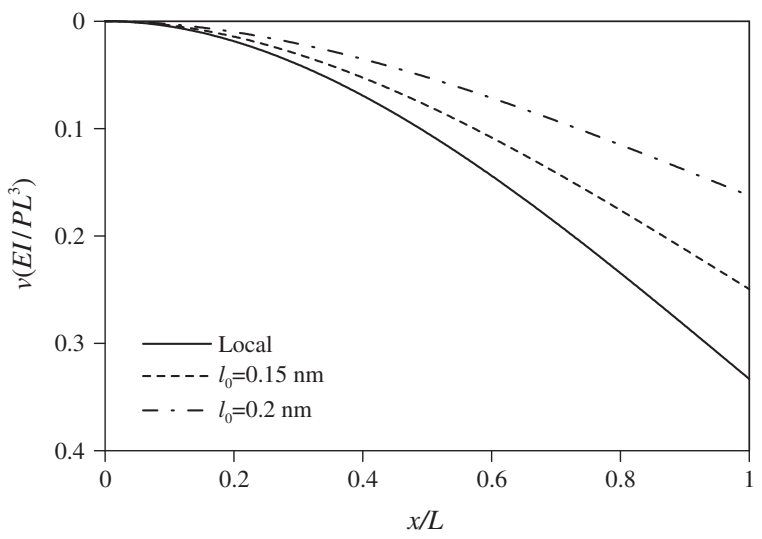

Fig. A.1. Non-dimensional deflection of a cantilever beam under tip load: comparison between the local and the proposed non-local solutions.

In Eqs. (15), $u_{i}, v_{i}$ and $\varphi_{i}(i=0, L)$ are given displacements and rotations at the beam ends. Appropriate initial conditions shall be supplemented to Eqs. (13).

The integro-differential Eqs. (13) along with the $\mathrm{BC}$ (15) govern the dynamics of the non-local TM beam. These are the most relevant features: (i) being the non-local effects modeled as internal body-forces, neither the mechanical $\mathrm{BC}$ nor the inertia forces are influenced by non-local effects; (ii) for a double-symmetric crosssection, axial and transverse responses are uncoupled; (iii) by suppressing the integral terms on the l.h.s. of Eqs. (13) and setting $\beta_{1}=1$, the motion equations of the classical local TM beam are retrieved.

\section{Numerical applications}

Next, the non-local TM beam theory is applied to the free vibration analysis of two beams with different BC: (i) a simply-supported beam; (ii) a cantilever beam. In both cases: a square cross-section with side length $h=1 \mathrm{~nm}$ is considered $\left(K_{s}=5 / 6\right)$; $L=5 \mathrm{~nm}$, while the other parameters are taken as those used by Reddy [5]. Further, in Eqs. (13) $\beta_{1}=1.0$ is set for the local terms, while the following exponential attenuation function is selected for the non-local terms:

$g(\mathbf{x}, \xi)=C \exp \left(-\frac{\|\mathbf{x}-\xi\|}{l_{0}}\right)$,

where $C$ is a constant and $l_{0}$ is the internal length, governing the maximum distance beyond which the long-range forces become 
negligible. In this paper, the values of $l_{0}$ and $C$ are set on a theoretical basis, to enhance non-local effects. For both the simply-supported and the cantilever beams $C=2 \times 10^{10}$ is assumed, while different values of $l_{0}$ are considered.

A few remarks are worth doing on the expected behavior of the proposed non-local TM beam. Being $\beta_{1}=1.0$ in Eqs. (13), the local part coincides with the classical local terms. On the other hand, the long-range forces are restoring forces due to a relative motion between volume elements, and introduce additional stiffness. As a result, it is expected that the proposed non-local solution is stiffer than the classical local one, that it progressively stiffens for an increasing $l_{0}$, to which corresponds indeed an increasing number of mutually interacting volume elements, and that it tends to the classical local solution for a decreasing internal length $l_{0}$, since the contribution of the non-local terms progressively vanishes (see Eq. (16)). In this respect, the proposed non-local beam model would reflect the experimental behavior of some materials [1,2].

For completeness, it is also noted that, based on the approach pursued in Ref. [12], a coefficient $\left(1-\beta_{1}\right)$ could be introduced in the constant $C$ governing the non-local interactions, as a complementary coefficient to $\beta_{1}$ scaling local effects in Eqs. (13), i.e. $\left(1-\beta_{1}\right)+\beta_{1}=1$. In this manner, the solution of the proposed non-local TM beam could be softer than the classical local one, depending on the choice of the parameters $\beta_{1}$ and $l_{0}$ [12]. In the authors' opinion, this should ensure a sufficient versatility to the proposed model, in view of different material behaviors to be fit, in the future, according to experimental evidence.

The eigenvalue problem associated to Eqs. (13) under the pertinent $\mathrm{BC}$ has been solved by resorting to a standard finite difference method with a uniform grid of $m+1$ points and $m$ intervals of amplitude $\Delta x=L / m(m=650)$. The integrals $I_{j}(x, \xi)$ in Eqs. (14), see Ref. [12], have been computed numerically based on a trapezoidal rule.

Fig. 2 shows the ratio $\omega^{(n l)} / \omega^{(l)}$ of the first three natural frequencies of the simply-supported non-local beam, $\omega^{(n l)}$, to the corresponding natural frequencies of the classical local beam, $\omega^{(l)}$, for different values of $l_{0}$. As expected, the non-local natural frequencies are larger than the corresponding local values and they all increase as $l_{0}$ increases. Fig. 3 displays the first three vibration modes of the simply-supported non-local beam, for two different values of $l_{0}$ along with the corresponding local mode shapes. The modes are symmetric or antisymmetric as in the local case, as a result of either a symmetric or an antisymmetric distribution of the longrange forces, as expected due to the symmetry of the BC. Further, in agreement with the results provided by different non-local beam theories [14], the non-local effect on the vibration modes of the simply-supported beam is not significant.

For the cantilever beam, Figs. 4 and 5 show the ratio $\omega^{(n l)} / \omega^{(l)}$ of the first three natural frequencies and the corresponding non-local and local modes. The non-local natural frequencies are larger than the local values and they all increase as $l_{0}$ increases. It can be also seen that, in this case, the mode shapes are considerably influenced by non-local effects due to the asymmetry of the BC.

\section{Conclusions}

The vibration analysis of a non-local beam model based on a mechanical approach to non-local elasticity has been pursued. Numerical applications have been presented where the natural frequencies of the non-local beam are larger than the corresponding ones of the classical local beam and increase with the internal length, due to a larger amount of long-range interactions in the beam. This behavior appears consistent with some experimental evidence in the literature $[1,2]$.

\section{Appendix A}

In this Appendix, the proposed non-local TM beam model is compared with the model developed by Peddieson et al. [15], based on the Eringen's non-local theory. The comparison focuses on the EB cantilever beam subjected to a static tip load $P$, for which the Peddieson's model does not predict non-local effects, although the flexural curvature field is linear [15]. Corrections to this paradox have been proposed based on alternative non-local constitutive laws [16].

For comparison, $\beta_{1}=1$ is set in Eqs. (13), the exponential attenuation function (16) is selected with $C=2 \times 10^{10}$ and different values of the internal length $l_{0}$; the geometrical and mechanical parameters chosen in Section 4 are used. The shear deformations are eliminated by setting $G A \rightarrow \infty$ in the local part of the TM beam model. Fig. A.1 shows that the (non-dimensional) displacement response of the cantilever beam as predicted by the proposed non-local model differs significantly from the classical local one. The non-locality follows from the fact that the proposed model is a displacement-based model, where the long-range forces are triggered by the relative displacements between volume elements of the beam due, in this case, to the tip load. The proposed nonlocal solution is stiffer than the classical local one, as expected since $\beta_{1}=1$ has been set in Eqs. (13) and due to the stiffening effect introduced by the long-range forces.

\section{References}

[1] D.C.C. Lam, F. Yang, A.C.M. Chong, J. Wang, P. Tong, J. Mech. Phys. Solids 51 (2003) 1477-1508.

[2] A.W. McFarland, J.S. Colton, J. Micromech. Microeng. 15 (2005) 1060-1067.

[3] L.F. Wang, H.Y. Hu, Phys. Rev. B 71 (2005) 195412-195418.

[4] P. Lu, H.P. Lee, C. Lu, P.Q. Zhang, Int. J. Solids Struct. 44 (2007) 5289-5300.

[5] J.N. Reddy, Int. J. Eng. Sci. 45 (2007) 288-307.

[6] Y.Y. Zhang, C.M. Wang, N. Challamel, J. Eng. Mech. 136 (5) (2010) 562-574.

[7] R.S. Lakes, J. Eng. Mater. Technol. 113 (1991) 148-155.

[8] S. Kong, S. Zhou, Z. Nie, K. Wang, Int. J. Eng. Sci. 46 (2008) 427-437.

[9] B. Wang, J. Zhao, S. Zhou, Eur. J. Mech. A/Solids 29 (2010) 591-599.

[10] M. Di Paola, G. Failla, M. Zingales, J. Elast. 97 (2009) 103-130.

[11] M. Di Paola, G. Failla, M. Zingales, Int. J. Solids Struct. 47 (2010) 2347-2358.

[12] M. Di Paola, G. Failla, A. Sofi, M. Zingales, Int. J. Mech. Sci. 53 (2011) 676-687.

[13] C. Polizzotto, Int. J. Solids Struct. 38 (2001) 7359-7380.

[14] M. Xu, Proc. R. Soc. A 462 (2006) 2977-2995.

[15] J. Peddieson, G.G. Buchanan, R.P. McNitt, Int. J. Eng. Sci. 41 (2003) 305-312.

[16] N. Challamel, C.M. Wang, Nanotechnology 19 (2008) 1-7. 345703. 